Acta Crystallographica Section E

Structure Reports

Online

ISSN 1600-5368

\title{
Redetermination of phenylhydrazinium chloride
}

\section{Geraldo M. de Lima, ${ }^{a}$ James L. Wardell ${ }^{a}$ and William T. A. Harrison $^{\text {b* }}$}

a'Departamento de Quimica, UFMG, Campus - Pampulha, 31270-901 Belo Horizonte, Minas Gerais, Brazil, and ${ }^{\mathbf{b}}$ Department of Chemistry, University of Aberdeen, Meston Walk, Aberdeen AB24 3UE, Scotland

Correspondence e-mail: w.harrison@abdn.ac.uk

Received 5 December 2007; accepted 14 December 2007

Key indicators: single-crystal X-ray study; $T=120 \mathrm{~K}$; mean $\sigma(\mathrm{C}-\mathrm{C})=0.007 \AA$; $R$ factor $=0.072 ; w R$ factor $=0.212 ;$ data-to-parameter ratio $=13.7$.

In the redetermined structure [Koo (1965). Bull. Chem. Soc. $J p n, 38,286]$ of the title compound, $\mathrm{C}_{6} \mathrm{H}_{9} \mathrm{~N}_{2}{ }^{+} \cdot \mathrm{Cl}^{-}$, the $\mathrm{H}$ atoms have been located and the hydrogen-bonding scheme established. A series of $\mathrm{N}-\mathrm{H} \cdots \mathrm{Cl}$ and $\mathrm{N}-\mathrm{H} \cdots \mathrm{N}$ hydrogen bonds leads to a layered network parallel to the (010) plane.

\section{Related literature}

For the earlier structure determination, see: Koo (1965). For a related structure, see: Hammerl et al. (2001). For reference structural data, see: Allen et al. (1987).

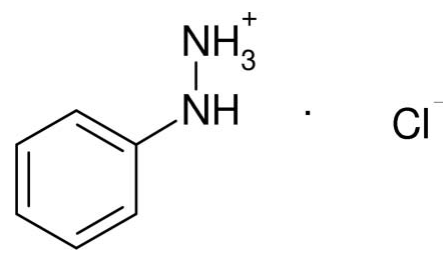

\section{Experimental}

\section{Crystal data}

$\mathrm{C}_{6} \mathrm{H}_{9} \mathrm{~N}_{2}{ }^{+} \cdot \mathrm{Cl}^{-}$

$M_{r}=144.60$

Monoclinic, $P 2_{1} / n$

$a=3.8223(5) \AA$

$b=30.461(5) \AA$

$c=6.0121(10) \AA$

$\beta=100.686(6)^{\circ}$

\section{Data collection}

Nonius KappaCCD diffractometer Absorption correction: multi-scan (SADABS; Bruker, 2003)

$T_{\min }=0.874, T_{\max }=0.995$

Refinement

$R\left[F^{2}>2 \sigma\left(F^{2}\right)\right]=0.072$

$w R\left(F^{2}\right)=0.212$

$S=1.12$

1288 reflections

94 parameters

Table 1

Hydrogen-bond geometry $\left(\AA{ }^{\circ}\right)$.

\begin{tabular}{lllll}
\hline$D-\mathrm{H} \cdots A$ & $D-\mathrm{H}$ & $\mathrm{H} \cdots A$ & $D \cdots A$ & $D-\mathrm{H} \cdots A$ \\
\hline $\mathrm{N} 2-\mathrm{H} 4 \cdots \mathrm{N} 1^{\mathrm{i}}$ & $0.93(6)$ & $2.11(6)$ & $3.031(6)$ & $173(5)$ \\
$\mathrm{N} 1-\mathrm{H} 1 \cdots \mathrm{Cl} 1^{\mathrm{ii}}$ & $0.90(6)$ & $2.49(6)$ & $3.256(4)$ & $142(5)$ \\
$\mathrm{N} 2-\mathrm{H} 2 \cdots \mathrm{Cl} 1^{\text {iii }}$ & $1.04(6)$ & $2.04(6)$ & $3.079(4)$ & $176(5)$ \\
$\mathrm{N} 2-\mathrm{H} 3 \cdots \mathrm{Cl} 1$ & $0.90(7)$ & $2.35(7)$ & $3.187(5)$ & $154(6)$ \\
\hline
\end{tabular}

Symmetry codes: (i) $x+1, y, z$; (ii) $x+1, y, z+1$; (iii) $x+\frac{1}{2},-y+\frac{1}{2}, z+\frac{1}{2}$.

Data collection: COLLECT (Nonius, 1998); cell refinement: SCALEPACK (Otwinowski \& Minor, 1997); data reduction: DENZO (Otwinowski \& Minor, 1997), SCALEPACK and SORTAV (Blessing, 1995); program(s) used to solve structure: SHELXS97 (Sheldrick, 1997); program(s) used to refine structure: SHELXL97 (Sheldrick, 1997); molecular graphics: ORTEP-3 (Farrugia, 1997); software used to prepare material for publication: SHELXL97.

The authors thank the EPSRC UK National Crystallography Service (University of Southampton) for the data collection.

Supplementary data and figures for this paper are available from the IUCr electronic archives (Reference: BT2663).

\section{References}

Allen, F. H., Kennard, O., Watson, D. G., Brammer, L., Orpen, A. G. \& Taylor, R. (1987). J. Chem. Soc. Perkin Trans. 1, pp. S1-19.

Blessing, R. H. (1995). Acta Cryst. A51, 33-38.

Bruker (2003). SADABS. Bruker AXS Inc., Madison, Wisconsin, USA.

Farrugia, L. J. (1997). J. Appl. Cryst. 30, 565.

Hammerl, A., Holl, G., Kaiser, M., Klapötke, T. M., Mayer, P., Nöth, H. \& Warchhold, M. (2001). Z. Anorg. Allg. Chem. 627, 1477-1482.

Koo, C. H. (1965). Bull. Chem. Soc. Jpn, 38, 286.

Nonius (1998). COLLECT. Nonius BV, Delft, The Netherlands.

Otwinowski, Z. \& Minor, W. (1997). Methods in Enzymology, Vol. 276, Macromolecular Crystallography, Part A, edited by C. W. Carter Jr \& R. M. Sweet, pp. 307-326. New York: Academic Press.

Sheldrick, G. M. (1997). SHELXS97 and SHELXL97. University of Göttingen, Germany. 


\section{supporting information}

Acta Cryst. (2008). E64, o330 [https://doi.org/10.1107/S1600536807066974]

\section{Redetermination of phenylhydrazinium chloride}

\section{Geraldo M. de Lima, James L. Wardell and William T. A. Harrison}

\section{S1. Comment}

The structure of the title compound, (I), was established by Koo (1965), but no hydrogen atoms were located. The hydrogen bonding pattern in the crystal of (I) cannot be established based on geometrical placement of the $\mathrm{H}$ atoms because roatation of the terminal $-\mathrm{NH}_{3}{ }^{+}$group leads to different plausible arrangements for the hydrogen bonds. Here, the redetermined structure of (I), including the $\mathrm{H}$ atom positions is presented (Fig. 1), and the hydrogen bonding scheme is definitively established.

In (I), atoms N1 and N2 deviate from the $\mathrm{C} 1-\mathrm{C} 6$ ring plane by -0.098 (4) $\AA$ and 0.418 (4) $\AA$, respectively. The bondangle sum for $\mathrm{N} 1$ is $331^{\circ}$, indicative of $s p^{3}$ hybridization for this atom. Otherwise, the geometrical paramaters for (I) may be regarded as normal (Allen et al., 1987).

The crystal packing for (I) is influenced by cation-to-cation $\mathrm{N}-\mathrm{H} \cdots \mathrm{N}$ and cation-to-anion $\mathrm{N}-\mathrm{H} \cdots \mathrm{Cl}$ hydrogen bonds (Table 1). The former of these leads to [100] chains in the crystal. One of the $\mathrm{H} \cdots \mathrm{Cl}$ separations is unusually short, with $\mathrm{H} \cdots \mathrm{Cl}=2.04$ (6) $\AA$, which possibly correlates with the its long $\mathrm{N}-\mathrm{H}$ separation of 1.04 (6) $\AA$. Together, the hydrogen bonds lead to sheets parallel to the (010) plane (Fig. 2).

Only one other crystal structure containing the phenylhydrazinium cation has been determined (Hammerl et al., 2001), which has similar geometrical paramaters to those in (I).

\section{S2. Experimental}

The title compound was prepared by dropwise addition of concentrated hydrochloric acid (1 equivalent) to an ethanolic solution of phenylhydrazine. The product, which appreared on standing, was collected and colourless blades of (I) were recrystallized from EtOH, m.p 525-528 K (decomp).

\section{S3. Refinement}

The N-bound $\mathrm{H}$ atoms were located in a difference map and their positions were freely refined with the constraint $U_{\text {iso }}(\mathrm{H})$ $=1.2 U_{\text {eq }}(\mathrm{N})$. The $\mathrm{C}$-bound $\mathrm{H}$ atoms were placed in calculated positions $(\mathrm{C}-\mathrm{H}=0.93 \AA)$ and refined as riding with $U_{\text {iso }}(\mathrm{H})=1.2 U_{\text {eq }}(\mathrm{C})$. 


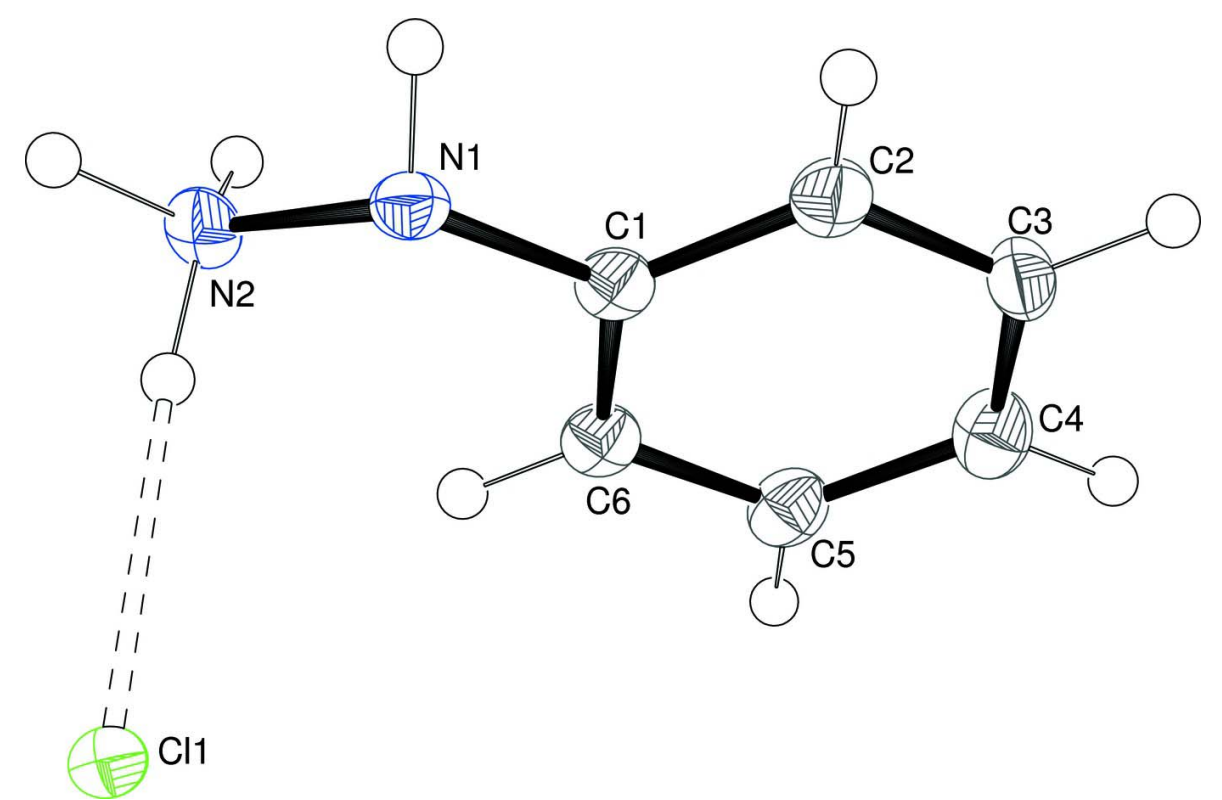

Figure 1

View of the molecular structure of (I) showing 50\% displacement ellipsoids. The $\mathrm{H}$ atoms are drawn as spheres of arbitrary radius and the hydrogen bond is shown as a double-dashed line.

phenylhydrazinium chloride

Crystal data

$\mathrm{C}_{6} \mathrm{H}_{9} \mathrm{~N}_{2}{ }^{+} \cdot \mathrm{Cl}^{-}$

$M_{r}=144.60$

Monoclinic, $P 2{ }_{1} / n$

Hall symbol: -P $2 \mathrm{yn}$

$a=3.8223(5) \AA$

$b=30.461(5) \AA$

$c=6.0121(10) \AA$

$\beta=100.686(6)^{\circ}$

$V=687.86(18) \AA^{3}$

$Z=4$

\section{Data collection}

Nonius KappaCCD

diffractometer

Radiation source: fine-focus sealed tube

Graphite monochromator

$\omega$ and $\varphi$ scans

Absorption correction: multi-scan

(SADABS; Bruker, 2003)

$T_{\text {min }}=0.874, T_{\max }=0.995$

\section{Refinement}

Refinement on $F^{2}$

Least-squares matrix: full

$R\left[F^{2}>2 \sigma\left(F^{2}\right)\right]=0.072$

$w R\left(F^{2}\right)=0.212$

$S=1.12$
$F(000)=304$

$D_{\mathrm{x}}=1.396 \mathrm{Mg} \mathrm{m}^{-3}$

Mo $K \alpha$ radiation, $\lambda=0.71073 \AA$

Cell parameters from 1173 reflections

$\theta=2.9-27.5^{\circ}$

$\mu=0.46 \mathrm{~mm}^{-1}$

$T=120 \mathrm{~K}$

Blade, colourless

$0.30 \times 0.07 \times 0.01 \mathrm{~mm}$

3891 measured reflections

1288 independent reflections

1073 reflections with $I>2 \sigma(I)$

$R_{\text {int }}=0.053$

$\theta_{\text {max }}=26.0^{\circ}, \theta_{\text {min }}=3.5^{\circ}$

$h=-4 \rightarrow 4$

$k=-35 \rightarrow 37$

$l=-6 \rightarrow 7$

1288 reflections

94 parameters

0 restraints

Primary atom site location: structure-invariant direct methods 
Secondary atom site location: difference Fourier map

Hydrogen site location: inferred from neighbouring sites

$\mathrm{H}$ atoms treated by a mixture of independent and constrained refinement

$$
\begin{aligned}
& w=1 /\left[\sigma^{2}\left(F_{\mathrm{o}}^{2}\right)+(0.0789 P)^{2}+3.5247 P\right] \\
& \text { where } P=\left(F_{\mathrm{o}}^{2}+2 F_{\mathrm{c}}^{2}\right) / 3 \\
& (\Delta / \sigma)_{\max }=0.001 \\
& \Delta \rho_{\max }=0.57 \text { e } \AA^{-3} \\
& \Delta \rho_{\min }=-0.62 \mathrm{e} \AA^{-3}
\end{aligned}
$$

\section{Special details}

Geometry. All e.s.d.'s (except the e.s.d. in the dihedral angle between two l.s. planes) are estimated using the full covariance matrix. The cell e.s.d.'s are taken into account individually in the estimation of e.s.d.'s in distances, angles and torsion angles; correlations between e.s.d.'s in cell parameters are only used when they are defined by crystal symmetry. An approximate (isotropic) treatment of cell e.s.d.'s is used for estimating e.s.d.'s involving 1.s. planes.

Refinement. Refinement of $F^{2}$ against ALL reflections. The weighted $R$-factor $w R$ and goodness of fit $S$ are based on $F^{2}$, conventional $R$-factors $R$ are based on $F$, with $F$ set to zero for negative $F^{2}$. The threshold expression of $F^{2}>\sigma\left(F^{2}\right)$ is used only for calculating $R$-factors(gt) etc. and is not relevant to the choice of reflections for refinement. $R$-factors based on $F^{2}$ are statistically about twice as large as those based on $F$, and $R$ - factors based on ALL data will be even larger.

Fractional atomic coordinates and isotropic or equivalent isotropic displacement parameters $\left(\AA^{2}\right)$

\begin{tabular}{lllll}
\hline & $x$ & $y$ & $z$ & $U_{\mathrm{iso}}^{*} / U_{\mathrm{eq}}$ \\
\hline $\mathrm{C} 1$ & $0.2381(11)$ & $0.12905(16)$ & $0.7787(9)$ & $0.0198(11)$ \\
$\mathrm{C} 2$ & $0.1470(12)$ & $0.10308(16)$ & $0.9478(9)$ & $0.0230(11)$ \\
H2A & 0.0795 & 0.1161 & 1.0771 & $0.028^{*}$ \\
C3 & $0.1557(13)$ & $0.05785(17)$ & $0.9259(9)$ & $0.0244(11)$ \\
H3A & 0.0939 & 0.0398 & 1.0416 & $0.029^{*}$ \\
C4 & $0.2530(13)$ & $0.03857(17)$ & $0.7383(9)$ & $0.0268(12)$ \\
H4A & 0.2574 & 0.0075 & 0.7245 & $0.032^{*}$ \\
C5 & $0.3432(13)$ & $0.06488(17)$ & $0.5721(9)$ & $0.0253(12)$ \\
H5 & 0.4120 & 0.0519 & 0.4432 & $0.030^{*}$ \\
C6 & $0.3349(12)$ & $0.11030(16)$ & $0.5904(9)$ & $0.0212(11)$ \\
H6 & 0.3955 & 0.1283 & 0.4741 & $0.025^{*}$ \\
N1 & $0.1991(10)$ & $0.17567(13)$ & $0.8023(7)$ & $0.0195(9)$ \\
H1 & $0.262(15)$ & $0.1852(18)$ & $0.947(11)$ & $0.023^{*}$ \\
N2 & $0.4198(11)$ & $0.20052(14)$ & $0.6761(8)$ & $0.0204(10)$ \\
H2 & $0.384(15)$ & $0.234(2)$ & $0.707(10)$ & $0.031^{*}$ \\
H3 & $0.303(17)$ & $0.1992(18)$ & $0.532(12)$ & $0.031^{*}$ \\
H4 & $0.652(17)$ & $0.190(2)$ & $0.712(10)$ & $0.031^{*}$ \\
C11 & $-0.2104(3)$ & $0.20115(4)$ & $0.2468(2)$ & $0.0192(4)$ \\
& & & & \\
\hline
\end{tabular}

Atomic displacement parameters $\left(\AA^{2}\right)$

\begin{tabular}{lllllll}
\hline & $U^{11}$ & $U^{22}$ & $U^{33}$ & $U^{12}$ & $U^{13}$ & $U^{23}$ \\
\hline $\mathrm{C} 1$ & $0.013(2)$ & $0.021(2)$ & $0.023(3)$ & $0.0028(17)$ & $-0.0015(19)$ & $0.0009(19)$ \\
$\mathrm{C} 2$ & $0.017(2)$ & $0.024(3)$ & $0.026(3)$ & $-0.0009(18)$ & $-0.002(2)$ & $0.002(2)$ \\
$\mathrm{C} 3$ & $0.024(3)$ & $0.025(3)$ & $0.024(3)$ & $-0.0042(19)$ & $0.003(2)$ & $0.007(2)$ \\
$\mathrm{C} 4$ & $0.026(3)$ & $0.021(3)$ & $0.034(3)$ & $0.003(2)$ & $0.006(2)$ & $0.001(2)$ \\
$\mathrm{C} 5$ & $0.021(3)$ & $0.025(3)$ & $0.030(3)$ & $-0.001(2)$ & $0.003(2)$ & $-0.002(2)$ \\
$\mathrm{C} 6$ & $0.014(2)$ & $0.024(2)$ & $0.025(3)$ & $0.0012(18)$ & $0.0011(19)$ & $0.000(2)$ \\
$\mathrm{N} 1$ & $0.018(2)$ & $0.022(2)$ & $0.017(2)$ & $-0.0013(15)$ & $0.0005(17)$ & $-0.0011(17)$ \\
$\mathrm{N} 2$ & $0.013(2)$ & $0.022(2)$ & $0.025(3)$ & $0.0040(16)$ & $-0.0003(17)$ & $0.0046(18)$
\end{tabular}




$\begin{array}{lllllll}\mathrm{C} 11 & 0.0187(6) & 0.0199(6) & 0.0182(7) & -0.0015(4) & 0.0015(4) & -0.0010(4)\end{array}$

Geometric parameters $\left(\AA,{ }^{\circ}\right)$

\begin{tabular}{|c|c|c|c|}
\hline $\mathrm{C} 1-\mathrm{C} 6$ & $1.378(7)$ & $\mathrm{C} 5-\mathrm{C} 6$ & $1.389(7)$ \\
\hline $\mathrm{C} 1-\mathrm{C} 2$ & $1.383(7)$ & $\mathrm{C} 5-\mathrm{H} 5$ & 0.9500 \\
\hline $\mathrm{C} 1-\mathrm{N} 1$ & $1.438(6)$ & $\mathrm{C} 6-\mathrm{H} 6$ & 0.9500 \\
\hline $\mathrm{C} 2-\mathrm{C} 3$ & $1.385(7)$ & $\mathrm{N} 1-\mathrm{N} 2$ & $1.449(6)$ \\
\hline $\mathrm{C} 2-\mathrm{H} 2 \mathrm{~A}$ & 0.9500 & $\mathrm{~N} 1-\mathrm{H} 1$ & $0.90(6)$ \\
\hline $\mathrm{C} 3-\mathrm{C} 4$ & $1.382(8)$ & $\mathrm{N} 2-\mathrm{H} 2$ & $1.04(6)$ \\
\hline $\mathrm{C} 3-\mathrm{H} 3 \mathrm{~A}$ & 0.9500 & $\mathrm{~N} 2-\mathrm{H} 3$ & $0.90(7)$ \\
\hline $\mathrm{C} 4-\mathrm{C} 5$ & $1.374(8)$ & $\mathrm{N} 2-\mathrm{H} 4$ & $0.93(6)$ \\
\hline $\mathrm{C} 4-\mathrm{H} 4 \mathrm{~A}$ & 0.9500 & & \\
\hline $\mathrm{C} 6-\mathrm{C} 1-\mathrm{C} 2$ & $120.6(5)$ & $\mathrm{C} 6-\mathrm{C} 5-\mathrm{H} 5$ & 119.6 \\
\hline $\mathrm{C} 6-\mathrm{C} 1-\mathrm{N} 1$ & $122.6(4)$ & $\mathrm{C} 1-\mathrm{C} 6-\mathrm{C} 5$ & $119.4(5)$ \\
\hline $\mathrm{C} 2-\mathrm{C} 1-\mathrm{N} 1$ & $116.6(4)$ & $\mathrm{C} 1-\mathrm{C} 6-\mathrm{H} 6$ & 120.3 \\
\hline $\mathrm{C} 1-\mathrm{C} 2-\mathrm{C} 3$ & $119.0(5)$ & $\mathrm{C} 5-\mathrm{C} 6-\mathrm{H} 6$ & 120.3 \\
\hline $\mathrm{C} 1-\mathrm{C} 2-\mathrm{H} 2 \mathrm{~A}$ & 120.5 & $\mathrm{C} 1-\mathrm{N} 1-\mathrm{N} 2$ & $112.5(4)$ \\
\hline $\mathrm{C} 3-\mathrm{C} 2-\mathrm{H} 2 \mathrm{~A}$ & 120.5 & $\mathrm{C} 1-\mathrm{N} 1-\mathrm{H} 1$ & $113(4)$ \\
\hline $\mathrm{C} 4-\mathrm{C} 3-\mathrm{C} 2$ & $121.0(5)$ & $\mathrm{N} 2-\mathrm{N} 1-\mathrm{H} 1$ & $105(4)$ \\
\hline $\mathrm{C} 4-\mathrm{C} 3-\mathrm{H} 3 \mathrm{~A}$ & 119.5 & $\mathrm{~N} 1-\mathrm{N} 2-\mathrm{H} 2$ & $108(3)$ \\
\hline $\mathrm{C} 2-\mathrm{C} 3-\mathrm{H} 3 \mathrm{~A}$ & 119.5 & $\mathrm{~N} 1-\mathrm{N} 2-\mathrm{H} 3$ & $104(4)$ \\
\hline $\mathrm{C} 5-\mathrm{C} 4-\mathrm{C} 3$ & $119.2(5)$ & $\mathrm{H} 2-\mathrm{N} 2-\mathrm{H} 3$ & $99(5)$ \\
\hline $\mathrm{C} 5-\mathrm{C} 4-\mathrm{H} 4 \mathrm{~A}$ & 120.4 & $\mathrm{~N} 1-\mathrm{N} 2-\mathrm{H} 4$ & $109(4)$ \\
\hline $\mathrm{C} 3-\mathrm{C} 4-\mathrm{H} 4 \mathrm{~A}$ & 120.4 & $\mathrm{H} 2-\mathrm{N} 2-\mathrm{H} 4$ & $116(5)$ \\
\hline $\mathrm{C} 4-\mathrm{C} 5-\mathrm{C} 6$ & $120.7(5)$ & $\mathrm{H} 3-\mathrm{N} 2-\mathrm{H} 4$ & $120(6)$ \\
\hline $\mathrm{C} 4-\mathrm{C} 5-\mathrm{H} 5$ & 119.6 & & \\
\hline $\mathrm{C} 6-\mathrm{C} 1-\mathrm{C} 2-\mathrm{C} 3$ & $0.2(7)$ & $\mathrm{C} 2-\mathrm{C} 1-\mathrm{C} 6-\mathrm{C} 5$ & $-0.5(7)$ \\
\hline $\mathrm{N} 1-\mathrm{C} 1-\mathrm{C} 2-\mathrm{C} 3$ & $175.6(4)$ & $\mathrm{N} 1-\mathrm{C} 1-\mathrm{C} 6-\mathrm{C} 5$ & $-175.5(4)$ \\
\hline $\mathrm{C} 1-\mathrm{C} 2-\mathrm{C} 3-\mathrm{C} 4$ & $-0.1(7)$ & $\mathrm{C} 4-\mathrm{C} 5-\mathrm{C} 6-\mathrm{C} 1$ & $0.6(7)$ \\
\hline $\mathrm{C} 2-\mathrm{C} 3-\mathrm{C} 4-\mathrm{C} 5$ & $0.2(7)$ & $\mathrm{C} 6-\mathrm{C} 1-\mathrm{N} 1-\mathrm{N} 2$ & $-27.3(6)$ \\
\hline $\mathrm{C} 3-\mathrm{C} 4-\mathrm{C} 5-\mathrm{C} 6$ & $-0.5(7)$ & $\mathrm{C} 2-\mathrm{C} 1-\mathrm{N} 1-\mathrm{N} 2$ & $157.5(4)$ \\
\hline
\end{tabular}

Hydrogen-bond geometry $\left(A,{ }^{\circ}\right)$

\begin{tabular}{lllll}
\hline$D-\mathrm{H} \cdots A$ & $D-\mathrm{H}$ & $\mathrm{H} \cdots A$ & $D \cdots A$ & $D-\mathrm{H} \cdots A$ \\
\hline $\mathrm{N} 2-\mathrm{H} 4 \cdots \mathrm{N} 1^{\mathrm{i}}$ & $0.93(6)$ & $2.11(6)$ & $3.031(6)$ & $173(5)$ \\
$\mathrm{N} 1-\mathrm{H} 1 \cdots \mathrm{C} 11^{1 i}$ & $0.90(6)$ & $2.49(6)$ & $3.256(4)$ & $142(5)$ \\
$\mathrm{N} 2-\mathrm{H} 2 \cdots \mathrm{C} 1{ }^{i i i}$ & $1.04(6)$ & $2.04(6)$ & $3.079(4)$ & $176(5)$ \\
$\mathrm{N} 2-\mathrm{H} 3 \cdots \mathrm{C} 11$ & $0.90(7)$ & $2.35(7)$ & $3.187(5)$ & $154(6)$
\end{tabular}

Symmetry codes: (i) $x+1, y, z$; (ii) $x+1, y, z+1$; (iii) $x+1 / 2,-y+1 / 2, z+1 / 2$. 